\title{
Analysis of the top 100 most influential papers in benign prostatic hyperplasia
}

\author{
Rohan Shah, BSc ${ }^{1}$; Jasmine Kashkoush, BSc ${ }^{1}$; Ahmed Kashkoush, BSc ${ }^{2}$; Trushar Patel, MD ${ }^{1}$ \\ ${ }^{1}$ Department of Urology, University of South Florida Morsani College of Medicine, Tampa, Florida, United \\ States; ${ }^{2}$ Department of Neurological Surgery, University of Pittsburgh Medical Center, Pittsburgh, Pennsylvania, \\ United States
}

Cite as: Can Urol Assoc J 2019 June 17; Epub ahead of print. http://dx.doi.org/10.5489/cuaj.5831

Published online June 17, 2019

$* * *$

\section{Abstract}

Introduction: The fund of knowledge on benign prostatic hypertrophy (BPH) has been growing since the 1970s. Citation analysis is a tool by which we can quantify influence of specific articles and assess the growth of a certain topic. This paper seeks to identify trends, as well as draw attention to the most influential papers, authors, and journals. Many analogous studies have been done, but none have been done in the field of BPH.

Methods: We used Thomson Reuters Web of Science to collect articles pertaining to BPH in a two-step fashion. We identified 117 keywords relevant to BPH and using these 117 words, we were able to identify 7302 total articles. These articles were organized by number of citations. Of the top 200 articles, 100 articles were excluded based on title and abstract analysis. One hundred articles were included for final analysis, as this is the standard of citation analysis.

Results: Overall, total citations were slightly correlated with journal impact factor. Author analysis revealed no significant difference between authorship and average citations. Topic analysis showed the most cited topic was surgical management with 657.35 citations per year. Study design analysis showed the predominant study design was the randomized control trial. Conclusions: By using the two-step methodology, we were able to create a list of the top 100 most influential articles in the field of BPH. In doing so, we illustrated the growth of the field over time and paid tribute to the myriad of papers, authors, and journals that have shaped the field to this day. 


\section{Introduction}

Benign prostatic hyperplasia (BPH) affects aging men worldwide, impacting a male's quality of life on a day-to-day basis. It is estimated that around $50-75 \%$ of men over 50 years of age and over $80 \%$ of men 70 years of age and older are affected by BPH. ${ }^{1}$ The hallmark of BPH is prostatic enlargement, which may lead to lower urinary tract symptoms (LUTS) including urinary frequency, nocturia, hesitancy, urgency, and incomplete emptying. ${ }^{2}$ Until recently, most of the epidemiologic information regarding $\mathrm{BPH}$ came from only men actively seeking treatment for $\mathrm{BPH}^{3}$ Since then, the wealth of knowledge surrounding the natural history, pathophysiology, diagnosis, and management of BPH has increased. ${ }^{3}$

Given the contributions made by countless researchers and physicians, a standard of care exists when diagnosing and managing BPH. Their efforts and dedication have aided symptomatic men, improving their quality of life in the process. Although there has been a rapid influx of information surrounding BPH, including updated screening guidelines and new approaches to surgical and medical management, it is crucial to recognize and highlight the seminal work that provided a foundation in how we now understand $\mathrm{BPH}$.

Citation analyses have been performed across different specialties to highlight the most influential papers on various topics. ${ }^{4-10}$ Measures such as the h-index and citation index (CI) help quantify an individual's research output and the number of times an article has been cited by other authors, respectively. ${ }^{11}$ In general, the more an article has been cited, the greater its impact within a field. ${ }^{11}$ The aim of this paper is to highlight the 100 most influential articles pertaining to BPH. We seek to shed light on these articles and their authors to highlight their impact on the field of urology. Furthermore, we seek to highlight advances within the field of urology pertaining to $\mathrm{BPH}$ to encourage researchers to continue their ongoing work.

In this study, we conducted a citation analysis using Thomson Reuters Web of Science to identify the 100 most influential articles on BPH. We did this to document the trends in research and highlight potential areas of deficiency that could also influence where research is directed in the future.

\section{Methods}

\section{Objective}

To identify and analyze the 100 most cited articles studying BPH cited between the years 1900 and 2018 in any journal. 100 papers were used as this is the convention in citation analyses.

\section{Inclusion criteria}

Studies were required to have focused on the pathophysiology, natural history, epidemiology, sequelae, diagnosis, or management of BPH. Papers that focused solely or predominantly on the study of prostate cancer, prostatism, LUTS, or erectile dysfunction were excluded. 


\section{Material}

We utilized all journals and databases contained within the Thomson Reuter's Web of Science in order to identify eligible studies.

\section{Data collection}

The literature query of the Thomson Reuters Web of Science was conducted in a two-step fashion (Figure 1).

During phase one of the data collection process, a topic search of the Web of Science databases was conducted with the keywords "benign prostatic hyperplasia.” These papers were then carefully reviewed to compile a list of keywords to be used during phase two of the data collection. These keywords were used to conduct a title search of the Web of Science database (Supplementary Table 1). The resulting 7300 articles were then sorted in descending order by total number of citations. Within the first 200 articles, the top 100 articles with the most citations that met the criteria outlined above were selected for final inclusion.

The following information was extracted from all 100 articles selected for final inclusion: title, author(s), journal of publication, year of publication, year of first citation, year of peak citation, total number of times cited, and number of times cited in 2018. Using this information, the average number of citations/year, the number of years until first citation, and the number of years until peak citation for each article was calculated.

Further analysis was conducted using each article's Altmetric score. Altmetric is a tool used to illustrate the attention that articles receive on the internet. It takes into account sources such as social media, traditional media, and blogs. Altmetric scores were extracted and analyzed for each paper.

\section{Results}

\section{Gross outcomes}

A total of 15965 articles were gathered from our database search, of which the top 100 most cited articles pertaining to BPH were extracted.

Figure 2 demonstrates the growth in the citation counts of the top 5 most cited articles since the year 1980. This mirrors the growth in citations overall. Of these five, only one article was published before 1992, titled, "The Development of Human Benign Prostatic Hyperplasia with Age” by Berry SJ et al. The total citations per article ranged from 732 to 2,042. The AUA Symptom Index experienced a sharp peak in citations from 2013 to 2015 which coincided with the peak of total overall citations on the subject of BPH in these 100 articles.

\section{Journal analysis}

Supplementary Table 2 lists the journals represented by articles included in the final list of 100 articles. It includes the Year of publication of the earliest article each journal had as well as the year of the latest article published per journal. Figure 3 provides a depiction of the number of 
average citations per journal. The highest contributor was The Journal of Urology with 200.67 average citations per year.

The articles that make up the top 100 came from several different journals with a few top contributors. As mentioned, the Journal of Urology was the journal with the highest number of citations per year. The Journal of Urology also had the most cited article on this list ("The American Urologic Association Symptom Index for Benign Prostatic Hyperplasia”). The journal with the highest impact factor with an article in this aggregate was the New England Journal of Medicine with an impact factor of 72.406 in 2016. The New England Journal of Medicine had 9 articles in the top 100 list. Figure 4 is a graph of the correlation between the average number of citations per year and impact factor of the top contributing journals. Included is a line of best fit $\left(y=0.1596 x+8.9486, R^{2}=0.225\right)$ indicating a slight positive correlation between impact factor and citations per year.

ANOVA analysis showed that there was no significant difference between average citation count across authors ( $\mathrm{p}=0.417$ ). Figure 5 displays the number of citations per author.

\section{Topic analysis}

Table 1 lists all of the topics focused on by these articles. The most common topic was diagnosis of BPH appearing in more than half of the publications on this list. The topic that yielded the highest number of average citations per year was surgical management at 657.35 citations per year.

Figure 6 depicts the number of citations per year for each of the topics. It shows a general increase in the number of citations per year for all topics with a peak within 2010-2015. However, the topics of diagnosis, natural history, epidemiology, and surgical management did not fit this trend. Diagnosis, in particular, trended upwards in the mid 1990s.

\section{Study design analysis}

Table 2 shows the types of study designs featured in these articles. The Randomized Control Trial was the most common study design utilized. 40 out of 100 of the articles were randomized control trials. The highest number of total citations also came from randomized control trials.

ANOVA analysis was done to determine if there was a statistically significant difference in total citation number between study design types $(\mathrm{p}=0.0008)$. ANOVA was also done to determine statistically significant difference between groups when comparing citations per year and was found to have a statistically significant difference $(p=0.000008)$.

\section{Altmetric analysis}

Altmetric score for all articles were obtained. There were 41 articles that had no detectable Altmetric score. The other 59 articles had scores that ranged from one to 141. Figure 7 is a scatter plot that correlates Altmetric score with citation count. There was no correlation found between Altmetric score and citation count. $(y=0.0184 x+1.4443$ 
$\left.\mathrm{R}^{2}=0.0895\right)$. Figure 8 shows Altmetric scores over time. There is a slight increase in Altmetric score with recency of publication.

\section{Discussion}

In this paper we have compiled a list of the most influential articles in the field of BPH. These 100 most influential articles have helped shape the field of knowledge of BPH since the inception of the term. In assembling this paper, we have created a list of works that depict our understanding of the diagnosis, treatment, and management of BPH. Among these works include articles which define the current modern standard of care. These seminal documents have influenced urologic practice for decades, providing a framework by which we can expect future research to build upon.

The first published article included in this list was Light Microscopic Stereological Analysis of the "Normal Human Prostate and of Benign Prostatic Hyperplasia" by Bartsch et al., which was first published in 1979 in the Journal of Urology and cited a total of 229 times. ${ }^{13}$ This was the earliest prominent article regarding BPH. In this study, the researchers analyzed prostate tissue from patients with BPH via light microscopy and compared them to normal prostate tissue. They found that BPH afflicted tissues had an increased volume of fibromuscular tissue and decreased glandular area, thus beginning the understanding of the condition from a scientific standpoint. This article helped sparked interest in the pathophysiology of the condition.

The most cited article was "The American-Urologic-Association Symptom Index of Benign Prostatic Hyperplasia” by Barry et al. published in 1992 in Journal of Urology. This article provided the framework by which the majority of American urologists evaluate BPH clinically by synthesizing known symptoms into an index of definitions and validating those definitions by testing their prevalence in patients with BPH. This index helps physicians meaningfully discriminate between patients with and without BPH by touching on all aspects of BPH including nocturia, hesitancy, urgency, dribbling, dysuria, and also takes into account how bothersome these symptoms are to the patient. ${ }^{15}$ In so doing, the "AUA Syptom Index of BPH" became a powerful tool for both practice and research protocols. It received a total of 2,042 citations since its publication and received about 75.63 citations per year. The influence of this article continues to grow as it received 94 citations in 2017.

Diagnosis was the most discussed topic on this list with a total of 53 total articles. This category included articles that described the criteria for diagnosing BPH. The top article in this field was The Effect of Finasteride in Med with Benign Prostatic Hyperplasia by Gormley et al. with 836 total citations. It was published in the New England Journal of Medicine in 1992. In this text Gormley details his double blind randomized control trial investigating the dosage of finasteride most effective in the treatment of $\mathrm{BPH} .{ }^{15}$ This is another example of the landmark texts that helped shape the practice of urology. Following the trend of change in citation growth from 1979 diagnosis there is a decline in the number of citations for the topic of diagnosis and instead an upward trend in articles discussing management and outcomes. Topics lacking in 
representation in this list included epidemiology, outcomes, symptomatology, medical management, and natural history. Given that these topics are very important in the management of patients afflicted with BPH research could be done to supplement this lacking in the future.

Altmetric scores were also investigated in this study. The website that gathers these scores provides many points of data on the online presence of each article. Overall no correlation was found between Altmetric score and citation count. This demonstrates that there's a divide between the public's valuation of articles about BPH and those of the scientific community. This could possibly illustrate the gap in understanding the public has of the scientific community and evidence based medicine in general. Aditionally, papers published later tended to have higher Altmetric scores (Figure 9). This could illustrate the public's preference for recency of literature over direct scientific value.

The method by which these articles were gathered aimed to isolate the most influential articles regarding BPH. Further analyses were performed to establish possible correlations between citations and various other factors. For example, journal impact factor was found to be slightly correlated with total citations. This was found to have a linear correlation when a linear regression was performed. Additionally, logarithmic regression was also performed to correlate rate of adoption of new articles. In many cases one-way ANOVA was also performed to confirm or refute statistically significant differences in total citations in the above-mentioned categories.

Several limitations in our study exist. First, a limitation of our study includes the nature of citation analyses. Citation analysis as a technique has been critiqued in the past as a form of assessing article influence. The most salient point of this critique focuses on whether number of citations is an accurate correlate of influence. This question stems from whether or not the assumptions made in this technique are valid. These assumptions include: that if a given article is cited it is implied that this article was used by the author, that a citation taken without context stands as equivalent in value to all citations, and that a cited document is related in context to a citing document. ${ }^{16}$ Inherently these assumptions are difficult to combat as it is impossible to quantify the importance of citation. However citation analysis is generally considered to be the best analogue for widespread influence. ${ }^{16}$

Other limitations of citation analysis include lack of measuring implicit citations, or citations . In this collection, we did not consider implicit citations as this information is not readily documented. Self-citations, or citations within an article of the same article, were also not taken into account. Conversely citation analysis as a technique has been lauded for its ability to quantify scientific usefulness. Given its ability to quantify data usage and its relatively low cost, citation analyses have become useful. It has also been viewed as a technique to grant larger exposure to health science trends. ${ }^{17}$ 


\section{Conclusions}

In this compilation we used the two-step methodology and careful review of each article to create a list of the 100 most influential articles in the field of benign prostatic hyperplasia. We found the most cited topic was surgical management, the most common study design was a randomized control trial, and that overall interest in BPH research has been highest in the past five years. In so doing we illustrated the evolution of scientific knowledge and the shifting change in our understanding of BPH, We identified potential areas of deficiency in topics to help pave the way for future works. We have also paid tribute to the authors, journals, and patients who have helped shape this field. 


\section{References}

1. Egan, K.B., The Epidemiology of Benign Prostatic Hyperplasia Associated with Lower Urinary Tract Symptoms: Prevalence and Incident Rates Urol Clin North Am, 2016. 43(3): p. 289-97.

2. Abdelmoteleb, H., E.R. Jefferies, and M.J. Drake, Assessment and management of male lower urinary tract symptoms (LUTS) Int J Surg, 2016. 25: p. 164-71.

3. Kirby, R.S., Textbook of benign prostatic hyperplasia 2nd ed. 2005, London: Taylor \& Francis. xiv, $570 \mathrm{p}$.

4. Coelho, D.H., L.W. Edelmayer, and J.E. Fenton, A century of citation classics in otolaryngology-head and neck surgery journals revisite. Laryngoscope, 2014. 124(6): p. 1358-62.

5. Fenton, J.E., Roy. D., Hughes, J.P., et al., A century of citation classics in otolaryngology-head and neck Surgery journals J Laryngol Otol, 2002. 116(7): p. 494-8.

6. Joyce, C.W., J.C. Kelly, and S.M. Carroll, The 100 top-cited classic papers in hand surgery J Plast Surg Hand Surg, 2014. 48(4): p. 227-33.

7. Kelly, J.C., Glynn, R.W., O'Brian, D.E., et al., The 100 classic papers of orthopaedic surgery: a bibliometric analysis J Bone Joint Surg Br, 2010. 92(10): p. 1338-43.

8. Murray, M.R., Wang, T., Schroeder, G.D., et al., The 100 most cited spine articles Eur Spine J, 2012. 21(10): p. 2059-69.

9. $\quad$ Ruegsegger, N., Ahmad, S.S., Benneker, L.M., et al., The 100 Most Influential Publications in Cervical Spine Research Spine (Phila Pa 1976), 2016. 41(6): p. 538-48.

10. Steinberger, J., Skovrij, B., Caridi, J.M., et al., The top 100 classic papers in lumbar spine surgery Spine (Phila Pa 1976), 2015. 40(10): p. 740-7.

11. Xu, J., et al., Citation Sentiment Analysis in Clinical Trial Papers AMIA Annu Symp Proc, 2015. 2015: p. 1334-41.

12. Roehrborn, C.G., McVary, K.T., Elion-Mboussa, A., et al., Tadalafil administered once daily for lower urinary tract symptoms secondary to benign prostatic hyperplasia: A dose finding study Journal of Urology, 2008. 180(4): p. 1228-34.

13. Bartsch, G., Muller, H.R., Oberholzer, M., et al., LIGHT MICROSCOPIC STEREOLOGICAL ANALYSIS OF THE NORMAL HUMAN-PROSTATE AND OF BENIGN PROSTATIC HYPERPLASIA Journal of Urology, 1979. 122(4): p. 487-91.

14. Coward, R.M. and C.C. Carson, Tadalafil in the treatment of erectile dysfunction Ther Clin Risk Manag, 2008. 4(6): p. 1315-30.

15. Gormley, G.J., et al., THE EFFECT OF FINASTERIDE IN MEN WITH BENIGN PROSTATIC HYPERPLASIA New England Journal of Medicine, 1992. 327(17): p. 118591.

16. Smith, L.C., Citation Analysis Library Trends, 1981. v30(1): p. 83-106.

17. Thompson, D.F. and C.K. Walker, A descriptive and historical review of bibliometrics with applications to medical sciences Pharmacotherapy, 2015. 35(6): p. 551-9. 
Figures and Tables

Fig. 1. Two-phase search.
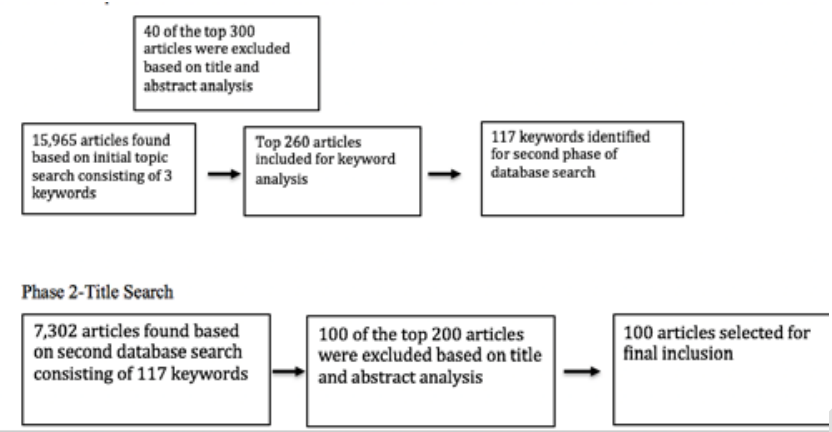

Fig. 2. Growth in citations.

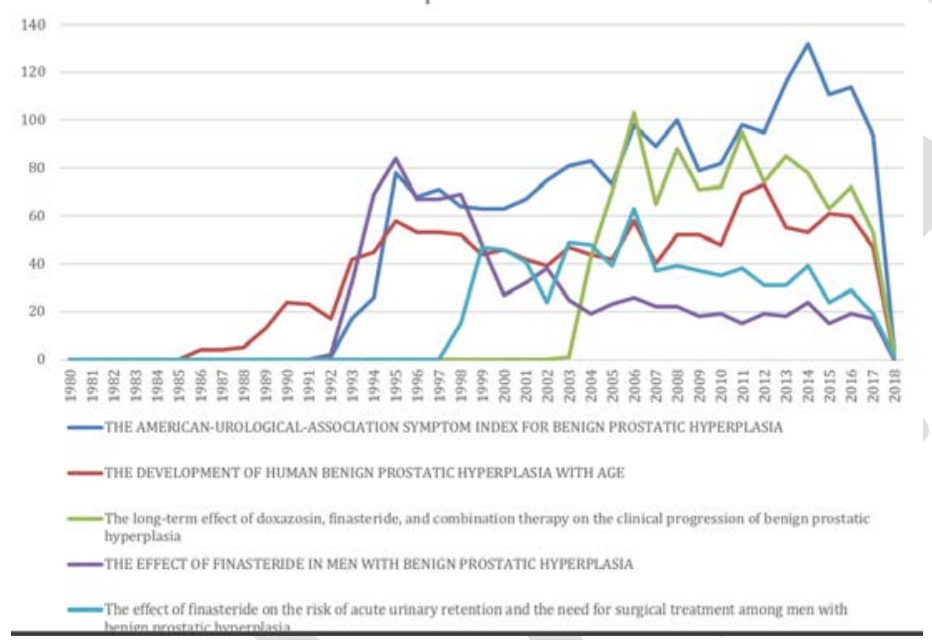


Fig. 3. Average citations/year/journal.

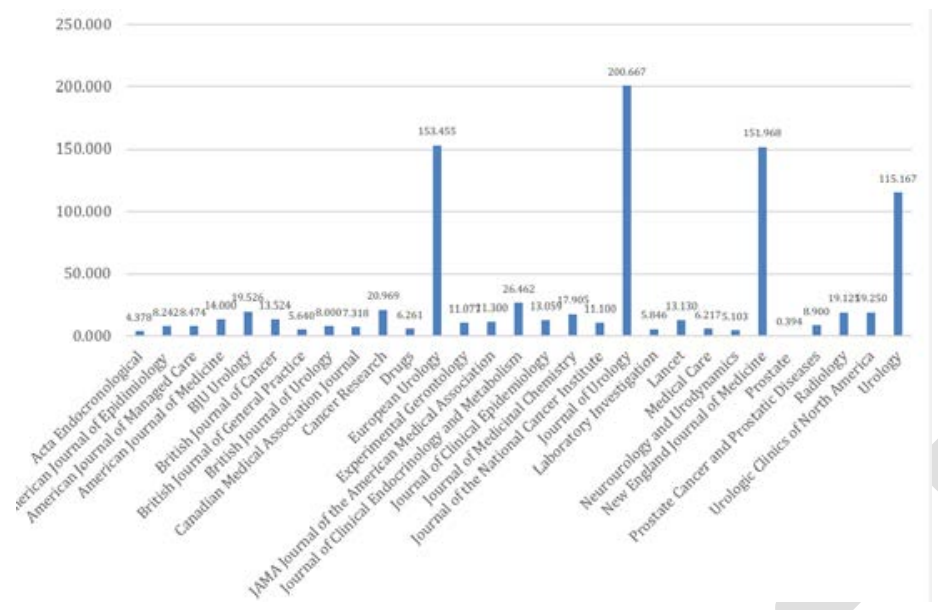

Fig. 4. Impact factor vs. citation.

Fig. 5. Citations/author/publication.

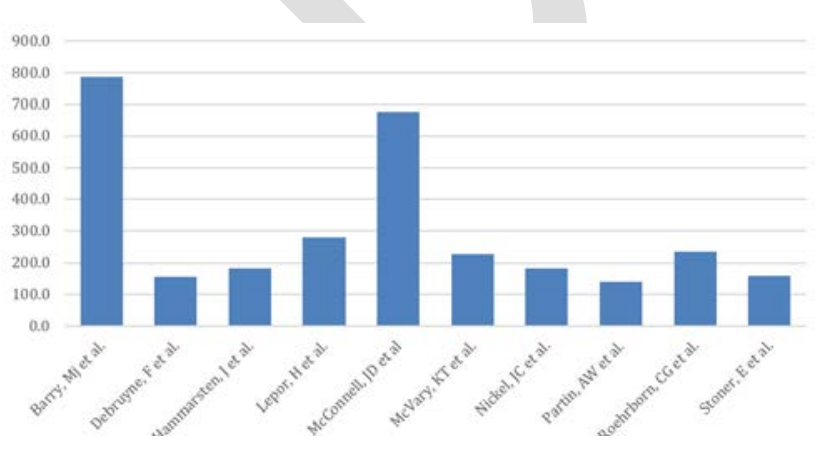


Fig. 6. Citations/year/topic.

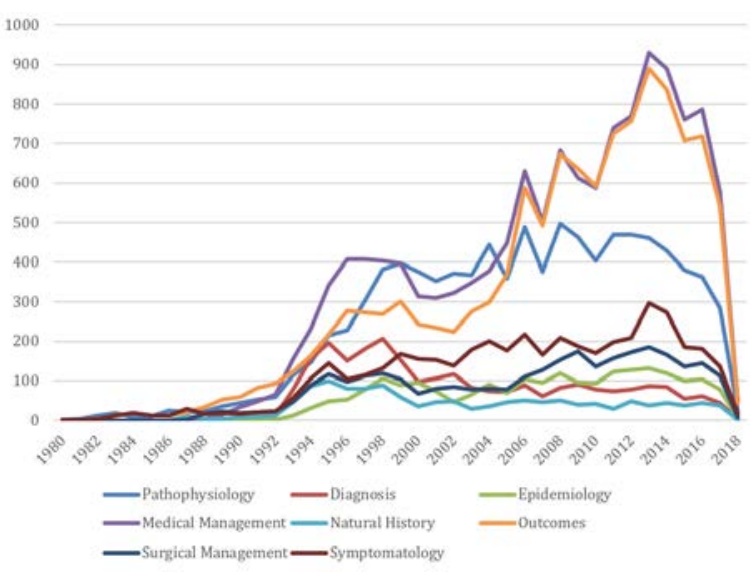

Fig. 7. Scatter plot that correlates Altmetric score with citation count.

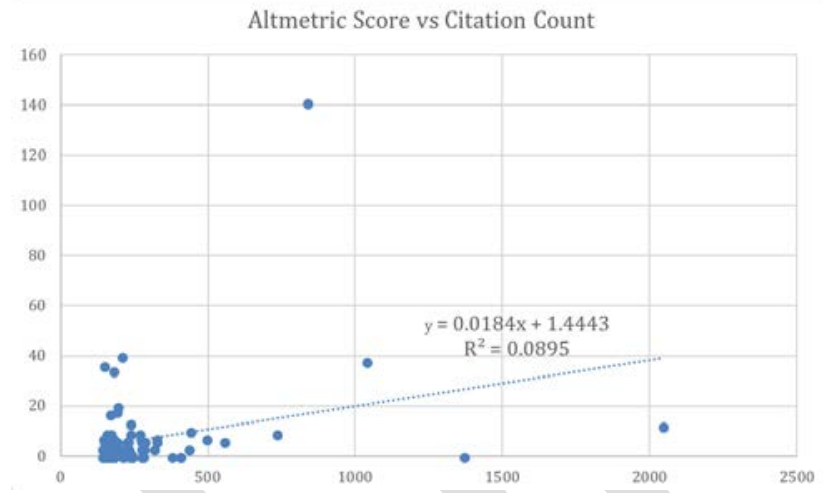

Fig. 8. Altmetric scores over time.

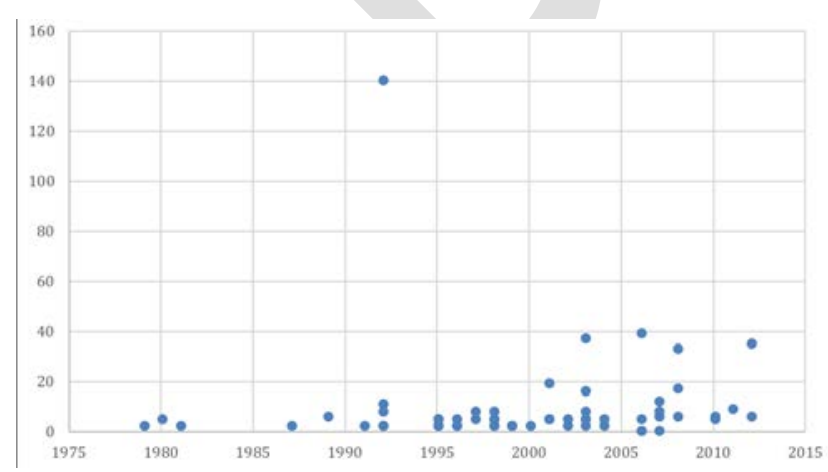


Fig. 9. Citation/year vs. number of years since publication.

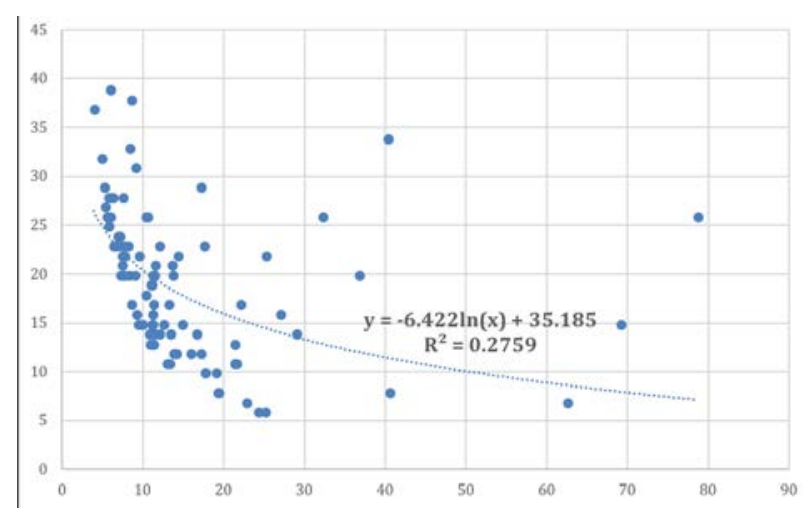

Table 1. Topics focused on by the top 100 articles

\begin{tabular}{|l|c|c|c|c|}
\hline Topic & $\begin{array}{c}\text { Number of } \\
\text { articles }\end{array}$ & Total citations & $\begin{array}{c}\text { Average } \\
\text { citations/year }\end{array}$ & Citations/article/year \\
\hline Diagnosis & 53 & 11440 & 408.57 & 19.03 \\
\hline Surgical management & 49 & 13147 & 657.35 & 12.91 \\
\hline Pathophysiology & 37 & 9475 & 242.95 & 12.31 \\
\hline Epidemiology & 18 & 4455 & 193.70 & 12.85 \\
\hline Outcomes & 12 & 3043 & 80.08 & 12.88 \\
\hline Symptomatology & 11 & 2161 & 108.05 & 20.02 \\
\hline Medical management & 5 & 2582 & 78.24 & 16.30 \\
\hline Natural history & 4 & 1335 & 41.72 & 5.23 \\
\hline
\end{tabular}

\begin{tabular}{|l|c|c|c|c|}
\hline Table 2. The types of study designs featured in the top 100 articles \\
\hline Study design & $\begin{array}{c}\text { Number of } \\
\text { articles }\end{array}$ & Total citations & $\begin{array}{c}\text { Average } \\
\text { citations/year }\end{array}$ & Citations/article/year \\
\hline Randomized control trial & 40 & 9927 & 342.31 & 15.07 \\
\hline Basic science & 13 & 2597 & 66.59 & 9.57 \\
\hline Cross-sectional study & 9 & 2639 & 77.62 & 13.29 \\
\hline Case control & 8 & 1517 & 47.41 & 8.07 \\
\hline Review & 8 & 1366 & 48.79 & 10.86 \\
\hline Systematic review & 8 & 1957 & 97.85 & 24.73 \\
\hline Prospective cohort study & 5 & 1236 & 37.45 & 11.33 \\
\hline Guidelines & 2 & 2196 & 84.46 & 42.94 \\
\hline
\end{tabular}




\begin{tabular}{|l|c|c|c|c|}
\hline Meta-analysis & 2 & 520 & 23.64 & 11.82 \\
\hline Parallel study & 2 & 499 & 49.90 & 28.99 \\
\hline Clinical trial & 1 & 279 & 9.00 & 9.00 \\
\hline Retrospective cohort study & 1 & 494 & 17.03 & 17.03 \\
\hline Survey & 1 & 239 & 11.38 & 11.38 \\
\hline
\end{tabular}

Supplementary Table 1. Keywords used to conduct a title search of the Web of Science database.

[To be added]

Supplementary Table 2. Journals represented by articles included in the list of top 100 articles

ACTA ENDOCRONOLOGICAL.

AMERICAN JOURNAL OF

EPIDIMIOLOGY

AMERICAN JOURNAL OF

RNAL OF

BNU UROLOGY

BRITISH JOURNAL OF CANCER

BRITISH JOURNAL OF GENERAL

PRACTICE

BRITISH JOURNAL OF UROLOGY

CANADIAN MEDICAL

ASSOCIATION JOURNAL

CANCER RESEARCH

DRUGS

EUROPEAN UROLOGY

EXPERIMENTAL GERONTOLOGY

JAMA JOURNAL OF THE

AMERICAN MEDICAL

ASSOCLATION

JOURNAL OF CLINICAI

ENDOCRINOLOCY

JOURNAL OF CLINICA

EPIDEMIOLOGY

JOURNAL OF MEDICINA

CHEMISTRY

JOURNAL OF THE NATIONAL

CANCER INSTITUTE

JOURNAL OF UROLOGY

LABORATORY INVESTIGATION

LANCET

MEDICAL CARE

NEUROUROLOGY AND

URODYNAMICS

NEW ENGLAND JOURNAL O

MEDICINE

PROSTATE

PROSTATE CANCER AND

PROSTATIC DISEASES

RADIOLOGY

UROLOGIC CLINICS OF NORTH

AMERICA

\begin{tabular}{|ll}
4.101 & 3.892 \\
5.595 & 7.496 \\
2.014 & 14.000 \\
5.514 & 8.474 \\
3.529 & 12.296 \\
5.908 & 13.524 \\
3.173 & 5.640 \\
1.69 & 8.000 \\
6.908 & 7.318 \\
9.826 & 10.841 \\
4.396 & 6.261 \\
13.994 & 17.274 \\
3.491 & 11.077 \\
38.209 & 11.300 \\
& \\
6.215 & 14.212 \\
& \\
4.978 & 13.059 \\
5.896 & 8.806 \\
14.537 & 11.100 \\
4.577 & 17.868 \\
4.401 & 5.846 \\
48.082 & 8.270 \\
3.508 & 6.217 \\
2.816 & 5.103 \\
64.201 & 25.680 \\
3.426 & 5.710 \\
3.283 & 8.900 \\
7.648 & 19.125 \\
1.597 & 6.417 \\
2.326 & 11.415 \\
& \\
& \\
& \\
& \\
&
\end{tabular}

$\begin{array}{llll}144 & 1981 & 1981 & 4.378\end{array}$

$\begin{array}{llll}434 & 1985 & 1994 & 8.242\end{array}$

$\begin{array}{llll}168 & 1980 & 1980 & 8.474\end{array}$

$\begin{array}{llll}322 & 2006 & 2006 & 14.000\end{array}$

$\begin{array}{llll}371 & 1999 & 2006 & 19.526\end{array}$

$\begin{array}{llll}284 & 1997 & 1997 & 13.524\end{array}$

$\begin{array}{llll}141 & 1993 & 1993 & 5.640\end{array}$

$\begin{array}{llll}184 & 1995 & 1995 & 8.000\end{array}$

$\begin{array}{llll}161 & 1996 & 1996 & 7.318\end{array}$

$\begin{array}{llll}671 & 1986 & 2001 & 20.969\end{array}$

$\begin{array}{llll}144 & 1995 & 1995 & 6.261\end{array}$

$\begin{array}{llll}3376 & 1996 & 2012 & 153.455\end{array}$

$\begin{array}{llll}144 & 2005 & 2005 & 11.077\end{array}$

$\begin{array}{llll}226 & 1998 & 1998 & 11.300\end{array}$

$\begin{array}{llll}688 & 1992 & 2006 & 26.462\end{array}$

$\begin{array}{llll}222 & 2001 & 2001 & 13.059\end{array}$

$\begin{array}{llll}339 & 1997 & 2000 & 17.905\end{array}$

$\begin{array}{llll}222 & 1998 & 1998 & 11.100\end{array}$

$\begin{array}{llll}7826 & 1979 & 2008 & 200.667\end{array}$

$\begin{array}{llll}152 & 1992 & 1992 & 5.846\end{array}$

$\begin{array}{llll}1502 & 1995 & 2003 & 13.130\end{array}$

$143 \quad 1995$

$148-1989$

$4711 \quad 1987$

$309-1990$

$178-199$

$153-2010$

$539-1990$

$2764 \quad 1994$

1994

6.217

5.103

151.968

0.394

8.900

19.125

19.250

115.167 